Esta es una versión postprint de: González-Albo, Borja, Moreno-Solano, Luz, Aparicio, Javier, Bordons, María (2018). "Contra-réplica a «Visibilidad de los institutos de investigación sanitaria a través de la base de datos Web of Science»". Revista Clínica Española, 218(4), 212-213.

La publicación final está disponible en https://doi.org/10.1016/j.rce.2018.01.009

\title{
Contra-réplica a «Visibilidad de los institutos de investigación sanitaria a través de la base de datos Web of Science»
}

Borja González-Albo ${ }_{1}$, Luz Moreno-Solano 2 , Javier Aparicio ${ }_{1}$, María Bordons 2

${ }_{1}$ Unidad Transversal de Apoyo a la Investigación (UTAI), Centro de Ciencias Humanas y Sociales (CCHS), Consejo Superior de Investigaciones Científicas (CSIC), Madrid, España

${ }_{2}$ Departamento de Ciencia, Tecnología y Sociedad. IFS, Centro de Ciencias Humanas y Sociales (CCHS), Consejo Superior de Investigaciones Científicas (CSIC), Madrid, España

Estimado Editor,

Quisiéramos agradecer el interés mostrado por Barredo et al.(1) hacia nuestro artículo(2), y contestamos a continuación a las tres reflexiones que plantean. 1) Coincidimos con dichos investigadores en que los datos de producción del trienio 2013-2015 son más consistentes que los de 2009-2011, pero se consideró importante analizar ambos periodos para detectar tendencias. Así, nuestro estudio concluye que no es viable identificar la contribución de los IIS a la investigación española a través de su presencia como institución firmante en las publicaciones recogidas por WoS, pero también evidencia un significativo aumento en la visibilidad de estos institutos, lo que constituye un hallazgo relevante. Por otro lado, aunque es cierto que los IIS de más reciente creación tienen menos posibilidades de aparecer como institución firmante en el primer periodo, en nuestro artículo se incluye la fecha de creación de todos los IIS con el fin de facilitar el análisis individualizado de los datos de cada instituto en función de su evolución histórica.2) Nos alegra saber que existe ya un documento consensuado sobre cómo referir la autoría en las publicaciones en el contexto de los IIS, y nos gustaría señalar la importancia de que su alcance sea amplio, no limitado a una sola comunidad autónoma, y bien aceptado por los investigadores, para que pueda contribuir a incrementar la visibilidad de los institutos y facilitar el seguimiento de su actividad en el futuro. 3) El análisis de la actividad de los grupos extra-hospitalarios no pudo abordarse en nuestro trabajo por su difícil identificación, ya que con frecuencia no incluyen al IIS de forma explícita en su afiliación. No obstante, consideramos que el estudio de estos grupos tiene un extraordinario interés, ya que desempeñan un papel crucial en el desarrollo de la investigación translacional, cuyo fomento fue una de las finalidades estratégicas que guiaron la creación de $\operatorname{los} I I S(3)$.

Referencias

(1) Barredo Torices, R., López Berlanga , M., Mier Barragán , I., Arenas Barbero, J. Réplica a: "Visibilidad de los institutos de investigación sanitaria a través de la base de datos Web of Science" . Revista Clínica Española 2018. 
(2) González-Albo, B. Moreno-Solano, L. Aparicio, J. Bordons, M. Visibility o healthcare research institute through the Web of Science database. $=$ Visibilidad de los institutos de investigación sanitaria a través de la base de datos Web of Science. En: Revista Clínica Española vol.217n.9 p. 510-517, 2017

(3) Real Decreto 339/2004, de 27 de febrero, sobre acreditación de institutos de investigación sanitaria. BOE num 63, 13 marzo 2004. 